This is a preprint. The final version will be available in Handbook of Sustainable Transport edited by Curtis, C., forthcoming 2020, Edward Elgar Publishing Ltd. The material cannot be used for any other purpose without further permission of the publisher, and is for private use only.

\title{
Is micro-mobility sustainable? An overview of implications for accessibility, air pollution, safety, physical activity and subjective wellbeing
}

\author{
Dimitris Milakis, Laura Gebhardt, Daniel Ehebrecht, Barbara Lenz \\ Institute of Transport Research, German Aerospace Center (DLR), Berlin, Germany
}

\begin{abstract}
Micro-mobility (MM) is usually presented as an environmental friendly, flexible and socially inclusive new group of travel modes. This chapter first defines and then explores the social sustainability potential of MM by providing an overview of MM implications for accessibility, air pollution, safety, physical activity and subjective wellbeing. Our results show that MM could enhance accessibility, yet costs, physical ability and technical skills might exclude certain social groups from using such modes. MM modes have no or limited direct $\mathrm{CO} 2$ emissions but environmental effects are uncertain if we account for the production of these modes. Road safety could be enhanced due to mutual awareness stimulation, but also compromised due to speed differential between $\mathrm{MM}$ and other modes. Physical activity could be increased or reduced depending on whether MM modes are electric or not. Exercising, enjoying the scenery, and having fun could enhance subjective well-being of MM mode users, while perceived safety risk could negatively influence subjective well-being. We conclude that MM sustainability potential should not be automatically assumed and could vary among the different MM modes. We suggest a set of regulatory actions with regards to permission, operation and monitoring of $\mathrm{MM}$ systems that could enhance their social sustainability.
\end{abstract}

Keywords: Micro-mobility implications, accessibility, air pollution, safety, physical activity, subjective wellbeing

\section{Introduction}

Micro-mobility (MM) is identified by popular media and scholarly literature as an emerging group of rather small-sized vehicles that could facilitate short-distance trips (mainly first- and last-mile trips) in a flexible, joyful and low-cost way (Tiwari 2019; Clewlow 2018; Kaufman and Buttenwieser 2018; Shaheen and Chan 2016). There is no clear definition of MM, but typically the term refers to vehicles that can carry one or two passengers, especially bicycles, various forms of scooters and small cars (and sometimes segways), often as part of docked and undocked sharing schemes that are based on mobility apps.

Micro-mobility is usually presented as a sustainable mobility solution, satisfying travelers' needs for flexibility, personalized mobility with a low environmental impact and high social inclusion potential (Hwang 2010; Semenov 2017). Micro-mobility modes might also have positive impacts on public transport use (Shaheen and Chan 2016). Yet, early evidence show that (a) e-scooter trips in New Zealand replace mainly trips that would have been undertaken by other sustainable modes such as walking, cycling or skateboard (Fitt and Curl 2019), (b) dockless MM solutions (e.g. bikes, scooters) can create safety conflicts with pedestrians and people with disabilities (Sikka et al. 2019), and (c) spatial availability of MM modes could be solely driven by demand and thus not equally distributed across the city (Smith, 2015; Mooney et al., 2019). 
This is a preprint. The final version will be available in Handbook of Sustainable Transport edited by Curtis, C., forthcoming 2020, Edward Elgar Publishing Ltd. The material cannot be used for any other purpose without further permission of the publisher, and is for private use only.

Thus far, the social sustainability (i.e. social inclusion and public health) potential of MM modes has not been systematically assessed in the literature. This chapter fills this gap by providing an overview of both positive and negative implications of MM for accessibility, air pollution, safety, physical activity and subjective wellbeing (SWB). Accessibility is a key factor for social inclusion (Lucas et al. 2015; Preston and Rajé 2007; van Wee and Geurs 2011) while air pollution, safety, physical activity (i.e. objective well-being) and subjective wellbeing are key factors for public health (van Wee and Ettema 2016).

The structure of the chapter is as follows. In section 2, we provide a definition of MM. In section 3, we present an overview of the implications of MM for accessibility (section 3.1), air pollution (section 3.2), safety (section 3.3), physical activity (section 3.4) and subjective wellbeing (section 3.5). The conclusions of our analysis are presented in section 4.

\section{Definition of micro-mobility}

Micro-mobility is a term often used to describe a group of modes that are typically electric, shared, hailed through an app and are used as "first/last mile" solution. Yet, there is still ambiguity about which modes can be included under this term. We suggest five categories of criteria according to which MM can be defined: functional, transport, passenger, use, and hailing (Table 1).

We identify a first group of criteria that a mode should meet to be included in the MM family (i.e. maximum speed of $45 \mathrm{~km} / \mathrm{h}$ and a maximum of two passengers) and a second group of criteria that differentiates the modes within the MM family: the size/volume, the motor/power (gas, electric, or pedal), foldability, the number of wheels, the parking (docked or dockless), the infrastructure (dedicated or shared), the position of the passengers (standing or sitting), whether passengers are active or passive during the trip, the use (private or shared, with or without driving licence) and the hailing method (smartphone, arm, voice or other) (Table 1).

\begin{tabular}{|c|c|c|c|c|}
\hline Functional & Transport & Passenger & Use & Hailing \\
\hline \multicolumn{5}{|c|}{ Inclusion criteria } \\
\hline $\begin{array}{c}\text { Speed } \\
\text { (ca. max.45 km/h) }\end{array}$ & & $\begin{array}{l}\text { Number } \\
(\max 2)\end{array}$ & & \\
\hline \multicolumn{5}{|c|}{ Differentiation criteria } \\
\hline Size/Volume & $\begin{array}{c}\text { Parking } \\
\text { (Docked/dockless) }\end{array}$ & $\begin{array}{c}\text { Position } \\
\text { (standing/sitting) }\end{array}$ & Private $(y / n)$ & Smartphone $(y / n)$ \\
\hline $\begin{array}{c}\text { Motor/Power } \\
\text { (gas/electric/pedal/push) }\end{array}$ & $\begin{array}{c}\text { Infrastructure } \\
\text { (Dedicated/shared) }\end{array}$ & Active/Passive & Shared $(y / n)$ & $\operatorname{Arm}(y / n)$ \\
\hline Folding $(\mathrm{y} / \mathrm{n})$ & & & $\begin{array}{l}\text { Driving licence } \\
\qquad(\mathrm{y} / \mathrm{n})\end{array}$ & Voice $(y / n)$ \\
\hline Wheels (1-4) & & & & Other \\
\hline
\end{tabular}

Table 1: The inclusion and differentiation criteria for MM modes.

Thus, we define MM as "the mobility performed by a vehicle with a maximum speed of $45 \mathrm{~km} / \mathrm{h}$ that can carry one or two persons". The threshold of $45 \mathrm{~km} / \mathrm{h}$ is based on the EU regulation (European Parliament and the Council of the European Union, 2013) that limits the speed of light passenger vehicles at $45 \mathrm{~km} / \mathrm{h}$ (including but not limited to those with an electric engine). 
This is a preprint. The final version will be available in Handbook of Sustainable Transport edited by Curtis, C., forthcoming 2020, Edward Elgar Publishing Ltd. The material cannot be used for any other purpose without further permission of the publisher, and is for private use only.

According to this definition, subcompact to large cars, motorcycles, rickshaws, motor scooters but also walking are excluded from the group of MM modes. The included modes can be distributed in three categories according to the vehicle size:

1. Large vehicles (e.g. Renault Twizy, OPEL RAK e; Ökoflitzer Paxter; AlkèATX 320E)

2. Medium vehicles (scooters: e-scooter, mini-scooter, elderly scooter; bicycles: E-bike, delivery bicycle, tandem; Segway)

3. Small vehicles (Personal Light Electric Vehicles (PLEV): (electric) skateboards, electric unicycles, self-balancing two-wheeled boards)

\section{Implications of micro-mobility for accessibility, air pollution, safety, physical activity and subjective wellbeing}

In the following sections, we present an overview of MM implications for accessibility, air pollution, safety, physical activity and subjective wellbeing based on existing literature and our analytical thinking. We identify both positive and negative implications of $M M$ in each field and distinguish implications per MM vehicle size whenever this is possible.

\subsection{Accessibility}

\subsubsection{Positive implications}

Small and medium MM modes could contribute in reaching destinations that are nearby in shorter time compared to walking or even in comparison to a car, when considering congestion (Noland 2019). MM modes can also be important for first-and-last-mile connectivity (Shaheen et al. 2012; Shaheen \& Chan 2016; Witzel 2018) (Table 2). For example, for various cities in China a number of studies suggest that dockless bike-sharing systems allow connecting to/from public transport (e.g. Du \& Cheng 2018; Gu et al. 2018). Similar implications apply for medium-sized cities in Canada and the US (Shaheen et al. 2012) and in Europe and Asia (Shaheen \& Chan 2016). The sharing company Lime (bikes and scooters) reported that $20 \%$ of its users mentioned to have used one of the company's bikes or e-scooters to access local stores and $40 \%$ to get to/from work or school in their last trip (Lime 2018). Medium-sized MM contribute to increasing mobility and hence participation in social life of less mobile user groups, such as elderly or physically disabled persons who are able to autonomously navigate a vehicle. Particularly e-bikes and specially designed escooters are relevant in this sense (Johnson 2018; May et al. 2010; Metz 2017; Fomiatti et al. 2013).

\subsubsection{Negative implications}

Large and medium MM modes might compete with public transport (Table 2). For example, the implementation of bike-sharing systems in some Canadian and US-American cities has led to a reduction in public bus usage (Shaheen et al. 2012). While large MM modes depend on (paved) road space, medium and small-sized MM modes also need specific infrastructure to some extent, such as paved grounds, allocated lanes, or parking facilities (e.g. docked shared mobility services) and therefore, cannot easily be used in all locations (e.g. Shaheen et al. 2010; Walker 2018). For less mobile persons, specifically, an increase of accessibility through medium MM vehicles is often limited by infrastructural impediments (Johnson 
This is a preprint. The final version will be available in Handbook of Sustainable Transport edited by Curtis, C., forthcoming 2020, Edward Elgar Publishing Ltd. The material cannot be used for any other purpose without further permission of the publisher, and is for private use only.

2018; May et al. 2010), such as missing elevators at public transport stations or adverse design of footpaths and lanes.

Accessibility gains of shared modes might become irrelevant if the services are docked and the distance to the next docking-station is too far (Gu et al. 2018). Dockless sharing systems have an advantage in this regard but only if a sufficient amount of vehicles is available in a respective area (Kaufman \& Buttenwieser 2018). Insights from a pilot programme for dockless bike-sharing in Seattle (Mooney et al. 2019) and from Chicago (Smith et al. 2015) show that the availability of vehicles, at least in the beginning, was better in areas with higher median incomes. A study on bike-sharing systems in Canadian cities showed that their users generally have higher education and higher incomes (Howorth \& Winters 2018). This leads to the question of affordability of MM modes since costs for purchasing or renting a $\mathrm{MM}$ vehicle can exclude poorer income groups. In this regard, MM could have a positive impact on accessibility only if costs are comparatively lower than other transport modes that would be used as an alternative. Moreover, some social groups might be excluded from using MM modes (Gu et al. 2018) as these in part require a certain level of physical ability, experience, technical adeptness, a driving licence and a credit card in the case of sharing services

An open question is also if and to what extent self-owned small and medium MM modes can be taken along when using public transport. This not only depends on local regulations and capacities of public transport but also on practicability. Foldable MM vehicles might ease this challenge to a certain degree (Zirn et al. 2018). Finally, the use of MM modes such as bikes and scooters depends on weather conditions, thus unfavourable conditions could limit their use and therefore reduce accessibility gains (Gu et al. 2018; Noland 2019).

\subsection{Air Pollution}

\subsubsection{Positive implications}

The key environmental benefit of MM modes is that they involve no or limited direct $\mathrm{CO} 2$ emissions when taking the 'tank to wheels' perspective (UN-Habitat 2013) and that they emit comparatively less other pollutants (Requia et al. 2018) (Table 2). Hwang (2010), for example, shows in his study on e-scooters in Taiwan that this MM mode has a positive impact on the reduction of $\mathrm{CO} 2$ emissions, when compared with scooters that have a combustion-engine. Additional environmental benefits could be derived by the replacement of car trip by MM modes. Early evidence suggest that this replacement rate is about $30 \%$ (Lime 2018; Fitt and Curl 2019). Moreover, shared MM can reduce vehicle use and vehicle kilometres travelled and thus emissions (Shaheen and Chan 2016) and energy use. This is related to the better overall efficiency of MM modes - they weigh less and use considerably less energy to move compared to larger vehicles, especially the car (Tillemann \& Feasley 2018).

\subsubsection{Negative implications}

The growth of MM implicates a growth in emissions and air pollution when considering the 'well-to-wheels' perspective. Indeed the production of electric MM involves the acquisition of resources for battery production, which can have negative environmental and social 
This is a preprint. The final version will be available in Handbook of Sustainable Transport edited by Curtis, C., forthcoming 2020, Edward Elgar Publishing Ltd. The material cannot be used for any other purpose without further permission of the publisher, and is for private use only.

effects (UN-Habitat 2013; González \& Prada 2015) (Table 2). Moreover, gains in direct emission reduction might be outweighed if fossil-based energy is used for the operation of MM vehicles (Hawkins et al. 2013; Klöckner et al. 2013; Semenov 2017). Likewise, LPGfuelled MM vehicles, such as some scooter models, also involve direct emissions when in use.

\subsection{Safety}

\subsubsection{Positive implications}

The emergence of MM could stimulate mutual awareness, respect and attention among road users (Table 2). Evidence from shared space environments show that road users have to rely on social rules and normative behaviour instead of traffic regulations (e.g. traffic signs or traffic lights). This requires road users to concentrate and be more attentive (to surrounding traffic/movement), which enhances traffic safety (Hamilton-Baillie, 2008).

\subsubsection{Negative implications}

E-scooters' relatively high speed could comprise pedestrians' safety, when they both share the sidewalk. The accident risk is increased for people with visual and hearing impairments, older (Sikka et al. 2019) and younger people. Early evidence from Santa Monica, California show that the severity of traffic accidents involving (standing) e-scooter users could vary from fractures and lacerations to head injuries, with younger users (below 18 years old) being more frequently involved in such accidents (Trivedi et al. 2019). Road safety could be further compromised by the increased speed of shared MM mode users trying to reduce costs, if usage is charged per minute. Also, the need to accomplish multiple tasks while using a MM mode (e.g. indicating turns by hand and keeping an eye on the overall traffic) could have adverse safety implications.

Small and medium size MM vehicles do not have any passive or active safety features (e.g. seatbelts, airbags) while the obligation to wear helmets varies among different countries resulting in significant safety deficiencies (see European Commission, 2013) (Table 2). While large MM vehicles, such as a twizy, offer more advanced safety features, they do not reach the safety level of cars, due to the smaller physical mass and lower weight (Gicklhorn et.al. 2019; Brost et al. 2019). The safety level requirements for MM vehicles differ between countries, as for instance, crash-tests for $\mathrm{MM}$ vehicles are regulated in some countries only (European Commission, 2013; Gicklhorn et al. 2019; Brost et al. 2019).

\subsection{Physical activity}

\subsubsection{Positive implications}

Non-electric MM modes (particularly cycling) could stimulate physical activity (Oja et al. 2011; Pucher et al. 2010). Especially in the case of car users, a switch to a combination of non-electric $\mathrm{MM}$ and public transport mode could significantly increase physical activity levels (Beirão \& Cabral, 2007) (Table 2). 
This is a preprint. The final version will be available in Handbook of Sustainable Transport edited by Curtis, C., forthcoming 2020, Edward Elgar Publishing Ltd. The material cannot be used for any other purpose without further permission of the publisher, and is for private use only.

\subsubsection{Negative implications}

Electric MM modes could replace trips previously undertaken by non-electric active MM modes (e.g. by bike or walking) inducing lower levels of physical activity (Hyvönen, Repo, \& Lammi, 2016) (Table 2). In particular, e-bike users tend to perceive that this mode increases their physical activity, yet they overall physical activity effect of switching to e-bike remains unclear (Jones et al. 2016). Moreover, early evidence from New Zealand indicates that 57\% of e-scooter trips replaced trips that would have been undertaken by active modes (e.g. foot, bicycle, e-bike, skateboard) (Fitt and Curl 2019).

\subsection{Subjective Wellbeing}

\subsubsection{Positive implications}

Small and medium MM modes can have a positive impact on the passengers' mood and subsequently on SWB by allowing them to enjoy the scenery (either urban or natural) and having fun riding the mode itself (Trainor et al., 2010; Stark et al., 2018) (Table 2).

For active MM modes (e.g. bicycle) physical activity can be an additional factor contributing to better mood, reduced anxiety and thus higher level of SWB (see e.g. Scully et al., 1998; Jones et al., 2016; Singleton, 2018). Small and medium MM modes could enhance interactions with other people thus directly improving passengers' mood or allow access to meaningful activities especially for those groups facing medical, physiological or financial restrictions in their mobility (Porcelli et al., 2014, Pettersson et al., 2016). Finally, small and medium MM modes could enhance the sense of autonomy in traveling. Singleton (2018) indicated that cyclists experience higher levels of autonomy compared to other mode users (e.g. public transport users).

For large MM modes, one source of positive mood could be the fun of driving. The level of autonomy and access to meaningful activities could be associated with positive well-being impacts as well.

\subsubsection{Negative implications}

MM modes (especially small- and medium-sized) could negatively influence mood and subsequently SWB because of increased perception of safety risk (Table 2). Singleton (2018) reported a significant positive association between cycling and fear. Higher perceived risk of cycling in a poor cycle-infrastructure context was identified in Greece (Milakis, 2015). The rather small range of small $\mathrm{MM}$ modes and therefore the need to perform a trip chain to reach a destination usually involving public transport could also influence negatively SWB. The lower levels of SWB for public transport compared to active mode users (e.g. cycling, walking) are well-reported in the literature (de Vos et al., 2013; Olsson et al., 2013). Likewise, the sense of autonomy (mainly) for small MM mode users would be in this case reduced, further influencing negatively their SWB. Finally, other factors that could increase commuting stress and thus reduce SWB are (a) road congestion for large MM modes because of reduced control over the trip (Novaco et al., 1990) and (b) sidewalk (or other infrastructure) crowding for small and medium MM modes because of reduced comfort (Vedel et al., 2017). 
This is a preprint. The final version will be available in Handbook of Sustainable Transport edited by Curtis, C., forthcoming 2020, Edward Elgar Publishing Ltd. The material cannot be used for any other purpose without further permission of the publisher, and is for private use only.

\begin{tabular}{|c|c|c|c|}
\hline \multicolumn{2}{|c|}{ Positive implications } & \multicolumn{2}{|c|}{ Negative implications } \\
\hline \multicolumn{4}{|c|}{ Accessibility } \\
\hline o & Access to areas that are not served by public transport & 0 & Competition with public transport \\
\hline ○ & Door-to-door trips (at least on shorter distances) & o & Contribution to traffic congestion \\
\hline o & First-and-last-mile connectivity & o & Cost-related exclusion \\
\hline ○ & Increased mobility of less mobile user groups (E-bikes, specially designed E-scooters) & ○ & Skills-related exclusion \\
\hline ○ & Increased mobility of persons without driving licence & & \\
\hline \multicolumn{4}{|c|}{ Air pollution } \\
\hline o & No direct emissions ( $\mathrm{CO} 2$, other pollutants; for electric and non-motorized modes) & o & Direct emissions, if gas-powered \\
\hline \multirow[t]{2}{*}{ ० } & Reduction of emissions by replacement of motorised trips & ○ & Emissions in production process \\
\hline & & 0 & Environmental and social effects of battery production (for electric modes) \\
\hline \multicolumn{4}{|r|}{ 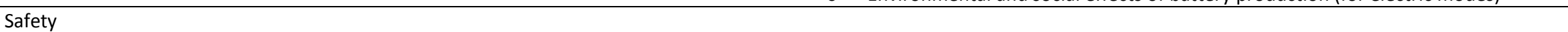 } \\
\hline \multirow[t]{3}{*}{ o } & Stimulation of mutual awareness among road users & o & Increased pedestrians' accident risk when sidewalk is shared with MM modes \\
\hline & & ० & Increased accidents' risk because of multitasking of MM users \\
\hline & & o & Increased accidents risk because of lack of regulation and of suitable infrastructure \\
\hline \multicolumn{4}{|c|}{ Physical activity } \\
\hline o & Stimulation of physical activity by non-electric MM modes & o & Replacement of active mode trips by electric MM modes \\
\hline \multicolumn{4}{|c|}{ Subjective wellbeing } \\
\hline o & Enjoyment of the scenery & 0 & Safety risk \\
\hline o & Travel fun & ० & Use of public transport in a trip chain (transfers, crowding, sense of captivity) \\
\hline o & Physical activity (only for MM active modes) & o & Sidewalk crowding \\
\hline ० & Sense of autonomy & o & Road congestion \\
\hline ० & Interactions with other people. & & \\
\hline ० & $\begin{array}{l}\text { Access to meaningful activities (mainly for mobility restricted populations, e.g. medical, } \\
\text { physiological or financial restrictions) }\end{array}$ & & \\
\hline
\end{tabular}

Table 2: Overview of negative and positive implications of MM for accessibility, air pollution, safety, physical activity, subjective wellbeing. 
This is a preprint. The final version will be available in Handbook of Sustainable Transport edited by Curtis, C., forthcoming 2020, Edward Elgar Publishing Ltd. The material cannot be used for any other purpose without further permission of the publisher, and is for private use only.

\section{Conclusions}

MM modes are usually presented as a sustainably mobility solution. Yet, there is no systematic assessment of this argument in the literature thus far. This chapter explored the social sustainability (i.e. social inclusion and public health) potential of MM by providing an overview of MM implications for accessibility, air pollution, safety, physical activity and subjective wellbeing. Below, we present a summary of our results per focal area followed by our conclusion.

MM modes have the potential to improve accessibility by enhancing first/last mile connectivity to public transport as well as by offering low-cost, seamless short distance trips. However, deployment of MM might have adverse effects on public transport use, at least on short distances. Moreover, involved costs, physical ability and technical skills might exclude certain social groups from using micro-mobility modes.

MM modes have no or limited direct $\mathrm{CO} 2$ emissions ('tank to wheels') and can further reduce emissions by replacing motorized trips. Environmental sustainability gains of electric $\mathrm{MM}$, however, depend on the type of energy used for its operation. Moreover, the production of $\mathrm{MM}$ vehicles - particularly the production of electric batteries and the resources needed in the process - might have negative effects on the environment and for the society ('well-to-wheels').

The emergence of $\mathrm{MM}$ implicates a diversification of existing modes. On the one hand, this might stimulate mutual awareness, respect and attention among road users enhancing road safety. On the other hand the mix of slower and faster moving MM modes increases the risk of traffic accidents.

Active MM modes such as bikes and non-electric scooters could stimulate physical activity. At the same time, walking or riding a bicycle could be replaced by the new electric MM modes reduce overall physical activity.

Active MM modes (e.g. bicycle, scooter, and skate) can have direct positive SWB effects by allowing travellers to exercise, enjoy the scenery, having fun with the mode itself, enhancing their autonomy and allowing participation to meaningful activities. SWB of small MM mode users could be negatively influenced by the increased perceived safety risk and fear, public transport trip chaining involving less autonomy and possible unpleasant interactions with other passengers, and sidewalk crowding reducing traveling comfort.

Our results show that both positive and negative implications for accessibility, air pollution, safety, physical activity and subjective wellbeing could follow the introduction of MM modes. Thus, we conclude that MM sustainability potential should not be automatically assumed and could vary among the different MM modes. We suggest that a set of regulatory measures with respect to permission, operation and monitoring of MM systems could help reduce potential adverse effects. Policies regarding permission of MM systems could involve vehicle safety standards, vehicle equipment and vehicle insurance. Policies regarding operation of MM systems could focus on infrastructure (e.g. specify by which MM modes and under which conditions sidewalks, cycle lanes, road lanes should be shared with other modes), accessibility (e.g. regulate distribution of fleets, pricing and non-digital access 
This is a preprint. The final version will be available in Handbook of Sustainable Transport edited by Curtis, C., forthcoming 2020, Edward Elgar Publishing Ltd. The material cannot be used for any other purpose without further permission of the publisher, and is for private use only.

for vulnerable populations and excluded areas), and environmental assessment (e.g. require lifecycle environmental assessment involving production and operation of $\mathrm{MM}$ fleets). Policies and plans regarding monitoring of the MM systems could focus on the fields and indicators to be observed (e.g. accessibility, safety), data to be acquired by the operating companies, surveys to be conducted in collaboration with the operating companies and actions to be taken according to the monitoring outcomes. Several European countries like Germany, Austria, Belgium, France, Norway, Finland, Sweden and Greece have taken the first regulatory steps regarding the operation (e.g. speed limits, rider's age, vehicle insurance, vehicle equipment, shared spaces) but not necessarily the accessibility and environmental aspects as well as the monitoring of MM systems. An unregulated, aggressive introduction of MM systems in cities could involve the risk of not realizing the full benefits of these systems, while at the same time decreasing the potential for further development because of a negative image to the public (e.g. due to safety incidences).

\section{References}

Beirão, G., \& Cabral, J. S. (2007). Understanding attitudes towards public transport and private car: A qualitative study. Transport Policy, 14 (6), 478-489.

Brost, M., Ewert, A., Eisenmann, C., Stieler, S. \& Gicklhorn, K. (2019). Elektrische Klein- und Leichtfahrzeuge - Mobilitätskonzepte mit Zukunftspotenzial? In: Journal für Mobilität und Verkehr, 2, 41- 49.

Clewlow, R. (2018), 'The micro-mobility revolution', accessed on 5 June 2019 at https://medium.com/populus-ai/the-micro-mobility-revolution-95e396db3754

De Vos, J., T. Schwanen, V. Van Acker and F. Witlox (2013), 'Travel and Subjective WellBeing: A Focus on Findings, Methods and Future Research Needs', Transport Reviews, 33 (4), 421-42.

$\mathrm{Du}, \mathrm{M}$. and Cheng, L. (2018), 'Better understanding the characteristics and influential factors of different travel patterns in free-floating bike sharing: evidence from Nanjing, China', Sustainability, 10 (4), 1244.

European Parliament and the European Council. Regulation (EU) No 168 (2013). On the approval and market surveillance of two- or three-wheel vehicles and quadricycles, 15 January 2013.

Fitt, H. and A. Curl (2019), E-Scooter Use in New Zealand: Insights around Some Frequently Asked Questions Key Points.

Fomiatti, R., J. Richmond, L. Moir and J. Millsteed (2013), 'A Systematic Review of the Impact of Powered Mobility Devices on Older Adults' Activity Engagement', Physical \& Occupational Therapy In Geriatrics, 31 (4), 297-309.

Gicklhorn, K., Ewert, A., Brost, M., Eisenmann, C. \& Stieler, S. (2019). Light Electric Vehicles in Baden-Württemberg - Potential for industry and new mobility solutions? 32nd Electric Vehicle Symposium (EVS32), 19.-22. Mai 2019, Lyon, Frankreich.

González, M. and de Prada, L. (2015), 'Environmental impacts of widespread shifting towards electricity based mobility', accessed on 5 June 2019 at http://www.greenemotionproject.eu/upload/pdf/deliverables/D9_5-Environmental-impacts-of-widespreadshifting-towards-electricity-based-mobility-V5_submitted.pdf

Gu, T., Kim, I. and Currie, G. (2019), 'To be or not to be dockless: empirical analysis of dockless bikeshare development in China', Transportation Research Part A, 119, 122147. 
This is a preprint. The final version will be available in Handbook of Sustainable Transport edited by Curtis, C., forthcoming 2020, Edward Elgar Publishing Ltd. The material cannot be used for any other purpose without further permission of the publisher, and is for private use only.

Hamilton-Baillie, B. (2008). Shared space: Reconciling people, places and traffic. Built environment, 34(2), 161-181.

Hosford, K. and Winters, M. (2018), 'Who are public bicycle share programs serving? An evaluation of the equity of spatial access to bicycle share service areas in Canadian cities', Transportation Research Record: Journal of the Transportation Research Board, 2672 (36), 42-50.

Hwang, J.J. (2010), 'Sustainable transport strategy for promoting zero-emission electric scooters in Taiwan', Renewable and Sustainable Energy Reviews, 14, 1390-1399.

Hyvönen, K., Repo, P., \& Lammi, M. (2016). Light electric vehicles: substitution and future uses. Transportation Research Procedia, 19, 258-268.

Johnson, R. (2018), 'Mobility scooters in the UK: public perception of their role', Proceedings of the Institution of Civil Engineers - Transport, 171 (4), 207-215.

Jones, T., Harms, L., \& Heinen, E. (2016). Motives, perceptions and experiences of electric bicycle owners and implications for health, wellbeing and mobility. Journal of Transport Geography, 53, 41-49.

Kaufman, S.M. and Buttenwieser, L. (2018), 'The state of scooter sharing in United States cities', $\quad$ accessed $\quad 5 \quad$ June 2019 at https://wagner.nyu.edu/files/faculty/publications/Rudin_ScooterShare_Aug2018_0.p df

Klöckner, C.A., Nayum, A. and Mehmetoglu, M. (2013), 'Positive and negative spillover effects from electric car purchase to car use', Transportation Research Part D: Transport and Environment, 21, 32-38.

Lime (2018), 'Year-end report 2018', accessed on 5 June 2019 at https://www.li.me/hubfs/Lime_Year-End\%20Report_2018.pdf

Lucas, K., B. van Wee and K. Maat (2015), 'A method to evaluate equitable accessibility: combining ethical theories and accessibility-based approaches', Transportation, 43 (3), 473-90.

May, E., Garrett, R. and Ballantyne, A. (2010), 'Being mobile: electric mobility scooters and their use by older people', Ageing \& Society, 30, 1219-1237.

Metz, D. (2017), 'Future transport technologies for an ageing society: practice and policy', Transport, Travel and Later Life, 10, 207-220.

Milakis, D. (2015), 'Will Greeks Cycle? Exploring Intention and Attitudes in the Case of the New Bicycle Network of Patras', International Journal of Sustainable Transportation, 9 (5), 321-34.

Mooney, S. J., K. Hosford, B. Howe, A. Yan, M. Winters, A. Bassok and J. A. Hirsch (2019), 'Freedom from the station: Spatial equity in access to dockless bike share', Journal of Transport Geography, 74, 91-6.

Noland, R.B. (2019), 'Trip patterns and revenue of shared e-scooters in Louisville, Kentucky', accessed on 5 June 2019 at https://transportfindings.org/article/7747-trip-patternsand-revenue-of-shared-e-scooters-in-louisville-kentucky

Novaco, R. W., D. Stokols and L. Milanesi (1990), 'Objective and Subjective Dimensions Of Travel Impedance as Determinants Of Commuting Stress', American Journal of Community Psychology, 18 (2), 231-57.

Oja, P., S. Titze, a. Bauman, B. de Geus, P. Krenn, B. Reger-Nash and T. Kohlberger (2011), 'Health benefits of cycling: A systematic review', Scandinavian Journal of Medicine and Science in Sports, 21 (4), 496-509.

Olsson, L. E., T. G??rling, D. Ettema, M. Friman and S. Fujii (2013), 'Happiness and Satisfaction with Work Commute', Social Indicators Research, 111 (1), 255-63. 
This is a preprint. The final version will be available in Handbook of Sustainable Transport edited by Curtis, C., forthcoming 2020, Edward Elgar Publishing Ltd. The material cannot be used for any other purpose without further permission of the publisher, and is for private use only.

Pettersson, I., L. Hagberg, C. Fredriksson and L. N. Hermansson (2016), 'The effect of powered scooters on activity, participation and quality of life in elderly users', Disability and Rehabilitation: Assistive Technology, 11 (7), 558-63.

Porcelli, P., M. Ungar, L. Liebenberg and N. Trépanier (2014), '(Micro)mobility, disability and resilience: exploring well-being among youth with physical disabilities', Disability and Society, 29 (6), 863-76.

Preston, J. and F. Rajé (2007), 'Accessibility, mobility and transport-related social exclusion', Journal of Transport Geography, 15 (3), 151-60.

Pucher, J., R. Buehler, D. R. Bassett and A. L. Dannenberg (2010), 'Walking and cycling to health: A comparative analysis of city, state, and international data', American Journal of Public Health, 100 (10), 1986-92.

Requia, W.J., Mohamed, M., Higgins, C.D., Arain, A. and Ferguson, M. (2018), 'How clean are electric vehicles? Evidence-based review of the effects of electric mobility on air pollutants, greenhouse gas emissions and human health', Atmospheric Environment, 185, 64-77.

Scully, D., J. Kremer, M. M. Meade, R. Graham and K. Dudgeon (1998), 'Physical exercise and psychological well being: A critical review', British Journal of Sports Medicine, 32 (2), 111-20.

Semenov, A. (2017), 'Why will micro mobility industry make the future?', accessed on 5 June 2019 at https://medium.com/@Splyt/why-will-micro-mobility-industry-make-thefuture-1b0a628ae3d0

Shaheen, S. and Chan, N. (2016), 'Mobility and the sharing economy: potential to facilitate the first- and last-mile public transit connections', Built Environment, 42 (4), 573-588.

Shaheen, S.A., Guzman, S. and Zhang, H. (2010), 'Bikesharing in Europe, the Americas, and Asia. Past, present, and future', Transportation Research Record: Journal of the Transportation Research Board, 2143 (1), 159-167.

Shaheen, S.A., Martin, E.W., Cohen, A.P. and Finson, R.S. (2012), 'Public bikesharing in North America: early operator and user understanding', accessed on 5 June 2019 at https://transweb.sjsu.edu/sites/default/files/1029-public-bikesharing-understandingearly-operators-users.pdf

Sikka, N., C. Vila, M. Stratton, M. Ghassemi and A. Pourmand (2019), 'Sharing the sidewalk: A case of E-scooter related pedestrian injury', The American Journal of Emergency Medicine, accessed at https://doi.org/10.1016/J.AJEM.2019.06.017.

Singleton, P. A. (2018), 'Walking (and cycling) to well-being: Modal and other determinants of subjective well-being during the commute', Travel Behaviour and Society, accessed at https://doi.org/10.1016/j.tbs.2018.02.005.

Smith, C.S., Oh, J.-S. and Lei, C. (2015), 'Exploring the equity dimensions of US bicycle sharing systems', accessed on 5 June 2019 at https://wmich.edu/sites/default/files/attachments/u428/2015/TRCLC_RR_14_01.pdf

Stark, J., M. Meschik, P. A. Singleton and B. Schützhofer (2018), 'Active school travel, attitudes and psychological well-being of children', Transportation Research Part F: Traffic Psychology and Behaviour, 56, 453-65.

Tillemann, L. and Feasley, L. (2018), 'Let's count the ways e-scooters could save the city', accessed on 5 June 2019 at https://www.wired.com/story/e-scooter-micromobilityinfographics-cost-emissions/

Tiwari, A. (2019), 'Micro-mobility: the next wave of urban transportation in India', accessed on 5 June 2019 at https://yourstory.com/journal/micro-mobility-edc6x8f1y1 
This is a preprint. The final version will be available in Handbook of Sustainable Transport edited by Curtis, C., forthcoming 2020, Edward Elgar Publishing Ltd. The material cannot be used for any other purpose without further permission of the publisher, and is for private use only.

Trainor, S., P. Delfabbro, S. Anderson and A. Winefield (2010), 'Leisure activities and adolescent psychological well-being', Journal of Adolescence, 33 (1), 173-86.

Trivedi, T. K., C. Liu, A. L. M. Antonio, N. Wheaton, V. Kreger, A. Yap, D. Schriger and J. G. Elmore (2019), 'Injuries Associated With Standing Electric Scooter Use', JAMA Network Open, 2 (1), e187381.

UN-Habitat (2013), 'Planning and design for sustainable urban mobility. Global report on human settlements 2013', New York et al.: Routledge.

van Wee, B. and D. Ettema (2016), 'Travel behaviour and health : A conceptual model and research agenda', Journal of Transport \& Health, 3, 240-8.

van Wee, B. and K. Geurs (2011), 'Discussing Equity and Social Exclusion in Accessibility Evaluations', European Journal of Transport and Infrastructure Research, 11 (11), 350-67.

Vedel, S. E., J. B. Jacobsen and H. Skov-Petersen (2017), 'Bicyclists' preferences for route characteristics and crowding in Copenhagen - A choice experiment study of commuters', Transportation Research Part A: Policy and Practice, 100, 53-64.

Walker, A. (2018), 'Don't ban scooters. Redesign streets', accessed on 5 June 2019 at https://www.curbed.com/word-on-the-street/2018/7/13/17246060/scooters-uberlyft-bird-lime-streets

Witzel, S. (2018), 'How micro mobility solves multiple problems in congested cities', accessed on 5 June 2019 at https://skedgo.com/how-micro-mobility-solves-multiple-problemsin-congested-cities/

Zirn, O., Sagert, K. and Rüther, M. (2018), 'Foldable electrified ultralight vehicles on public walkways for sustainable traffic chains', International Journal of Civil Infrastructure, $1,1-8$. 